\title{
Ion tracking in photocathode rf guns
}

\author{
John W. Lewellen \\ Advanced Proton Source, Argonne National Laboratory, Argonne, Illinois 60439
}

(Received 20 September 2001; published 19 February 2002)

\begin{abstract}
Projected next-generation linac-based light sources, such as PERL or the TESLA free-electron laser, generally assume, as essential components of their injector complexes, long-pulse photocathode rf electron guns. These guns, due to their design rf pulse durations of many milliseconds to continuous wave, may be more susceptible to ion bombardment damage of their cathodes than conventional rf guns, which typically use rf pulses of microsecond duration. This paper explores this possibility in terms of ion propagation within the gun, and presents a basis for future study of the subject.
\end{abstract}

DOI: $10.1103 /$ PhysRevSTAB.5.020101

PACS numbers: 41.75.Ht, 41.75.Fr, 29.25.Bx

\section{INTRODUCTION}

Current designs for next-generation linac-based light sources, whether they are proposed as dedicated machines (e.g., PERL [1]) or as parts of other high-energy accelerators [e.g., the TESLA free-electron laser (FEL) [2] ], tend to have certain common design features. The proposed linacs are usually either entirely or mostly superconducting in order to allow long bunch trains and high duty-factor operation. Average beam currents tend to be high, on the order of $0.1-1 \mathrm{~A}$. The $\mathrm{rf}$ frequency is typically $L$-band $(1.3 \mathrm{GHz})$ as a compromise between cavity construction concerns and projected performance.

Naturally, the injectors for these proposed machines are typically also $L$-band (although there are exceptions, e.g., a proposed subharmonic gun based on the Boeing highduty-factor gun [3] for PERL). High quantum efficiency (QE) cathodes are also taken to be essential parts of the injector design, given the laser power requirements for copper, magnesium, or other low-QE materials. Typical high-QE cathodes, however, have generally been found to be much more sensitive to environmental conditions, such as residual gases in the gun, than lower-QE materials [3-6].

In general, no cavity can be rendered completely free of residual gases. Therefore, the passage of the electron beam through the photocathode rf gun can result in ionization of the residual atmosphere. This is true for any photoinjector. However, for the photoinjectors envisioned for future light sources, there are several important differences from those commonly used today. First, these guns are to be operated at high accelerating gradients on time scales ranging from milliseconds to true continuous wave operation, as opposed to a few microseconds for more conventional guns. Second, rather than generating a single electron bunch, these guns are anticipated to produce long-duration bunch trains by placing electron bunches in a large fraction of the available rf "buckets." In the case of PERL, for instance, it is anticipated that every bucket may in fact be filled. The result is the continuous creation of ions and the continuing presence of strong fields, which may cause the ions to mi- grate in a preferred direction inside the gun. In de guns, in general any (positive) ion created will backstream to the cathode. In rf guns the situation is more complex due to the rapidly time-varying rf fields.

The intent of this paper is not to predict in detail what affects ion bombardment would have on the operation of a $\mathrm{cw}$ photoinjector, the lifetime of its cathode, or the quality of the electron beam generated by the gun. ${ }^{1}$ Rather, this work seeks to address two more fundamental questions. First, how will the ions, once formed, move under the influence of the cw rf fields within the gun? Second, if ions are found to move towards the region of the cathode, can some estimate be made of the possible impact energy and distribution at the cathode region? As an auxiliary question, are existing rf gun simulation tools adequate to answer these questions?

Thus, this paper explores the possibility of ion motion and propagation in a photoinjector, given $\mathrm{cw}$ operation, and generates estimates of ion impact energy and distribution on the cathode region of the gun, assuming a few typical species, water and hydrogen, found in many accelerator systems. (Both water and hydrogen are commonly found in relatively large quantities in operating accelerators, at least during initial conditioning when one might expect much of the cathode damage to occur, and there is a large enough mass difference between the two that simulating these two species should give an idea of how effects scale with species charge/mass ratio.) One of the standard photoinjector simulation codes, PARMELA, is used for this study, and its advantages and limitations for this application are noted.

\footnotetext{
${ }^{1}$ For further information regarding ion damage to highquantum efficiency GaAs photocathodes, Ref. [4] is a reasonable starting point although the gun in question is a dc gun. Some interesting quantum efficiency effects with CsTe cathodes in a relatively long-pulse duration $\mathrm{rf}$ gun are described in Ref. [5]. Reference [3] contains some lifetime information for a long-pulse, extremely high-QE rf gun.
} 


\section{SIMULATION METHODOLOGY AND PARAMETERS}

\section{A. Initial assumptions}

Several assumptions are used throughout this initial study.

First, the source atoms or molecules are assumed to be uniformly distributed within the volume of the gun. They are assumed to be converted to ions only from impact by the electron beam. Ionization from dark current, secondary emission electrons, the incoming photocathode drive laser pulse, or the rf field itself is neglected.

Second, no multiple ionization events are assumed to take place. Similarly, after the initial "generation" (i.e., start of the simulation) no ions are assumed to become deionized through electron capture. This is the least justifiable assumption; however, given the limits of the simulation code used, it is one that is effectively required.

Third, ion-ion interactions are not included. The validity of this assumption will be verified later.

Fourth, instead of simulating the passage of every electron bunch in a photoinjector, a continuous, steady-state on-axis charge distribution may be substituted. The validity of this assumption will be addressed later.

Finally, the ions are assumed not to be strongly affected by either the magnetic field of the focusing solenoid typically located at or close to the output port of many photoinjector designs or by the magnetic field of the electron beam [7]. In the simulations performed to date, the maximum ion kinetic energy (for hydrogen) corresponds to a velocity of about $0.5 \%$ of the speed of light, so this is a reasonable assumption. The rf magnetic field is applied by the simulation code as a matter of course.

\section{B. Simulation model}

The basic design of the gun used in the simulation is a fairly traditional SLAC/BNL/UCLA-type 1.5-cell cavity with a resonant frequency of $1.3 \mathrm{GHz}$. The detailed gun geometry was provided by Manoel Conde of the Argonne Wakefield Accelerator (AWA) group at Argonne National Laboratory. The POISSON/SUPERFISH codes [8] were used to generate the required fields from the model geometry, shown in Fig. 1.

The simulation code PARMELA $[9,10]$ was used to perform the actual particle tracking. PARMELA is very frequently used for photocathode rf gun studies and is considered a standard code in the field. It includes some features (such as independent particle placement within the body of the gun) that make it convenient for this work; unfortunately, the lack of a good method of changing a particle's charge "on the fly" limits this study to the single-ionization assumptions listed above. The model PARMELA uses for space charge should be valid for regimes of particle motion encountered in this problem. The simulations were generally run with time steps of 1 degree of $L$-band phase

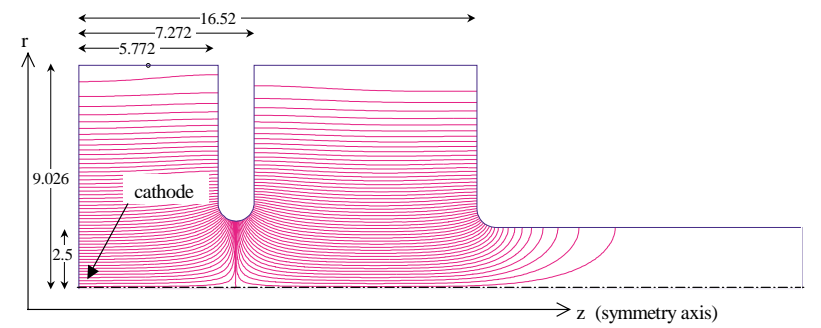

FIG. 1. (Color) Basic geometry of the model gun used in these simulations. The rf electric field lines are shown; the gun is operating in a $\pi$ mode. The gun is cylindrically symmetric about the $z$ axis. The length of the beam is sufficient to ensure that boundary conditions at the end of the tube do not affect either the rf field pattern or the gun's resonant frequency. All dimensions are in centimeters; further details of the construction should be obtained from the Argonne Wakefield Accelerator staff.

(approximately $2.14 \mathrm{ps}$ ); selected runs were performed using shorter time steps to verify that the 1 degree increment provides reasonable accuracy. Ion particle locations were recorded at regular intervals during the tracking. For PARMELA aficionados, the only unusual feature used was to reverse the rf field in the gun, so that the exit plane of the problem was the cathode plane of the gun. The ion tracks are identical in both cases (taking into account, of course, the change in $z$-coordinate direction and sign due to the field reflection).

In order to determine the appropriate starting phase of the rf field in the gun, a reference electron bunch was propagated from the cathode, with a launch phase of 35 degrees following the zero crossing, and average gun gradient of $34.5 \mathrm{MV} / \mathrm{m}$; this provides for an exit electron beam energy of $7.3 \mathrm{MeV}$ and represents a typical operational gradient for the AWA gun used as the simulation model [11]. The reference particle flight path is used to determine the phase of the rf field when the electron beam reaches a given longitudinal position within the gun. For a given longitudinal coordinate, ion starting locations are specified via an external file. Assuming that the initial gas species have uniform density in the gun, the ion macroparticle radial coordinates are chosen so as to place the particles in the center of equal-area rings. Thus, assuming an equal probability of ion creation, each ion macroparticle represents an equal number of actual ions.

Once the simulation is started, the ion macroparticles are tracked until all have either impacted a wall or the simulation time expires. No multiple ionizations or deionizations are assumed to take place while the ions are in motion. In the case where the gun may be used for single-bunch operation, i.e., during tune-up, this is a reasonable assumption. When operating bunch trains, however, there is a definite possibility of multiple ionization events for species other than hydrogen, and electron capture and deionization is also always a possibility. These effects are beyond the current capabilities of PARMELA, but could be included in a purpose-written code. 
If the simulation assumes single-bunch operation there is no field from an on-axis electron beam to influence the ion motion. In multibunch operation, however, there is a pulsed on-axis electron beam, the fields of which can influence the ion motion. The effects of these fields are approximated in the simulation by including the field from an on-axis charged cylinder, with charge uniformly distributed along the length of the gun, a radius of $1 \mathrm{~mm}$, and a charge density of $1.36 \mathrm{nC} / \mathrm{cm}^{3}$, such that the total on-axis charge contains $1 \mathrm{nC}$ along the distance traveled by an electron bunch in one rf period. Since the ions move slowly compared to the electron beam, this distribution, in effect, simulates the field averaging the ions would see as many bunches are generated and transit the gun; in effect, this includes only the zeroth harmonic of the beam electric fields. This method does not include the effects of the electron beam magnetic field; however, as mentioned above, these should be negligible. Also, the electron beam transverse size will change as the beam moves through the gun; this is also not taken into consideration. The generated electrostatic potential is shown in Fig. 2.

The justification for effectively ignoring the higher harmonics of the beam field is as follows. One may estimate the fields from the beam by considering a point charge moving along a straight line and then, for a periodic beam (e.g., every rf bucket filled), obtain the time harmonics of the field at a defined point in space; the magnitude of the harmonics can then be compared to the strength of the rf field at that location. At a typical point from which ions may propagate to impact on the cathode, for example, $10 \mathrm{~cm}$ from the cathode longitudinally and $0.5 \mathrm{~cm}$ in radius, the ratios are on the order of $9 \times 10^{-5}: 1$ in the longitudinal direction and $6 \times 10^{-3}: 1$ in the radial direction. In addition, the time average of the longitudinal beam fields is, to a reasonable first approximation, zero; the time average of the radial beam field is always such that the ions would be drawn towards the axis, and the sum of the radial field harmonics is equivalent to the dc field used in the model. The dc field model used does, as shown in Fig. 2, contain nonzero longitudinal electrostatic field components due to the interior structure of the gun. Thus, time averaged over many rf periods, the electrostatic field as represented by the on-axis charge distribution should be a reasonable first model of the beam fields.

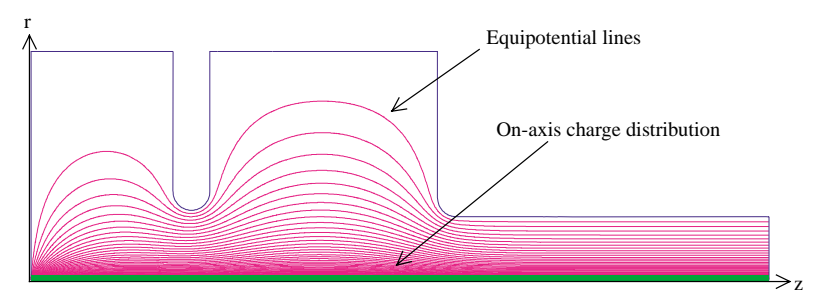

FIG. 2. (Color) Equipotentials in the gun generated via an on-axis uniform charge distribution. The radius of the assumed charge distribution is $1 \mathrm{~mm}$.
The particular ion species chosen for study were $\mathrm{H}^{+}$ ions and $\mathrm{H}_{2} \mathrm{O}^{+}$. Both hydrogen and water are found in relatively large quantities during initial pumpdown and conditioning in many accelerators. The point for these simulations, however, is not the particular species, but rather the assumed charge/mass ratio of the ion species. If the results for $\mathrm{H}^{+}$and $\mathrm{H}_{2} \mathrm{O}^{+}$are similar and reasonable, for instance, then one might expect to find $\mathrm{CH}_{4}{ }^{+}$ions, also commonly found in accelerators, behaving similarly.

Finally, the simulation ignores the starting motion of the ions, e.g., they are assumed to start at rest instead of a velocity taken from some distribution based on the average thermal velocity. The validity of this assumption was verified by imposing an appropriate velocity distribution on the input beam and noting that this had little to no discernible impact on the ion flight tracks. Since the fields in the gun are symmetric, ions are placed out only along one axis (typically $+x$ or $+y$ ).

\section{Simulation results $-\mathrm{H}^{+}$ions}

We begin by considering what happens to hydrogen ions starting at a specific longitudinal coordinate and at varying radii and ignore for the moment whether a particular ion macroparticle would be within the electron beam radius at that longitudinal position. The use of hydrogen eliminates the multiple ionization assumption. Hydrogen may be derived from any number of molecular species such as methane, water, ethanol, etc., so assuming its presence in a gun is probably reasonable. Ion starting positions are chosen with a longitudinal spacing of $0.5 \mathrm{~cm}$, from 0.5 to $17.0 \mathrm{~cm}$ from the cathode. Radial starting positions and starting phases are selected as described above.

Some sample ion tracks for hydrogen atoms are shown in Fig. 3. Note that, depending on the starting location (and thus time), the ions can have very different flight paths through the gun. Some starting locations have ions directed towards the cathode in a broad spray, some result in ions being trapped between the cathode and full cell, and some result in well-collimated streams of ions being either ejected from the gun or directed towards the cathode. Figure 4 combines data from plots such as those shown in Fig. 3 from all of the launch positions into an impact energy plot. The $(z, r)$ location in the plot shows the assumed ion starting location, and the color of the point represents the impact energy at the cathode plane. For these plots, a single-shot electron beam is assumed, e.g., no effects of an on-axis electron beam are included. Figure 5 is an impact energy plot for hydrogen including the effects of an on-axis electric charge on ion propagation, as described above, assuming a bunch charge of $1 \mathrm{nC}$ and every bucket filled. There are differences due to the on-axis fields, but the overall distributions are very similar and the peak impact energies are also nearly the same. One aspect of the appearance of these plots deserves mention in order to avoid confusion. In order to generate a visible spot with 


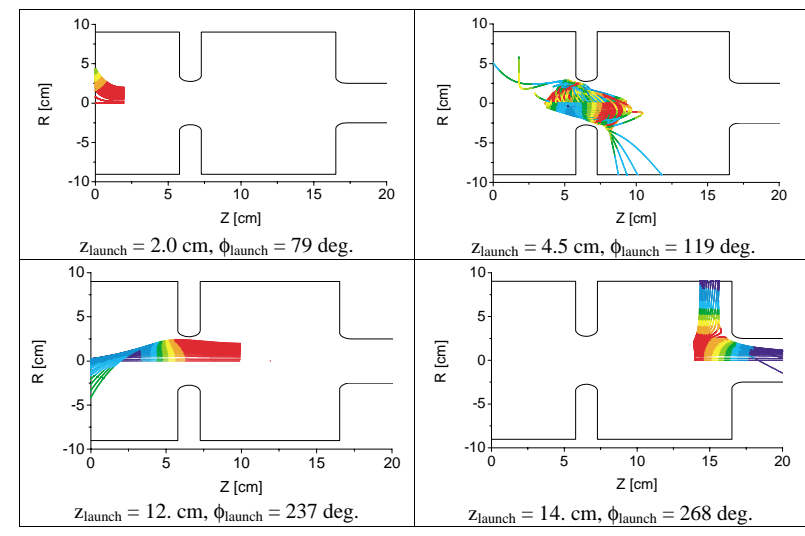

FIG. 3. (Color) Hydrogen ion trajectories for four different longitudinal starting coordinates, and no electron beam field. Trajectories are color coded to ion energy (red is the minimum, blue is the maximum for each individual plot). $Z_{\text {launch }}$ and $\phi_{\text {launch }}$ are, respectively, the starting longitudinal coordinate and starting phase for the ions (e.g., $\phi_{\text {launch }}$ is the phase at which the centroid of a reference electron beam bunch reaches $\left.z_{\text {launch }}\right)$.

discernible color, a square patch is used instead of a single dot to represent the ion starting locations. Because the radial starting coordinates are chosen so as to allow each macroparticle to represent an equal-area ring, as described above, the squares overlap in the radial dimension.

The $\mathrm{H}^{+}$ion tracks and impact energies show very strong dependence upon the time and location of ionization. The $\mathrm{H}^{+}$ions that impact the cathode plane were found to gain kinetic energies of up to almost $13 \mathrm{kV}$, or about $4 \times 10^{5}$ the average thermal energy (for room-temperature cavities). The potential wells formed by the electron beam, in contrast, are on the order of $300 \mathrm{~V}$ at most, for 1-nC charge per bucket and every bucket filled. Therefore, it is not surprising that the presence of the on-axis charge distribution does not generate a significant shift in the impact-energy plots in terms of final impact energy from a given starting location and phase. There is a noticeable difference on the ion trajectories, however, which also shows up as a strong influence on the ion impact distribution over the cathode plane.

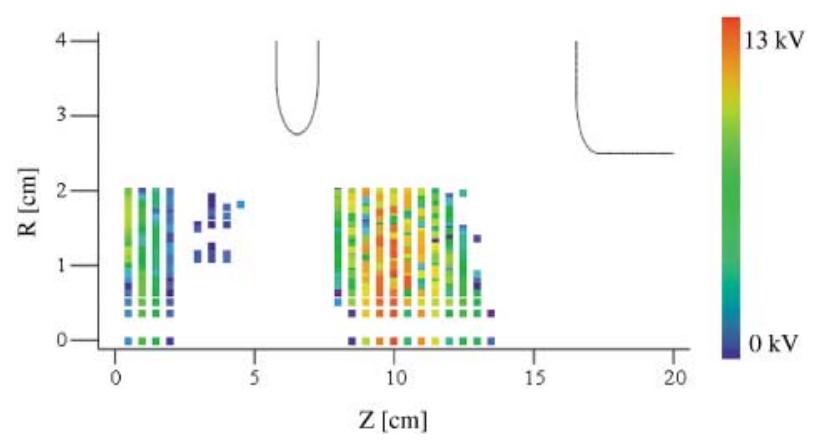

FIG. 4. (Color) Impact energy at the cathode plane as a function of starting location, for $\mathrm{H}^{+}$ions. Red indicates the highest impact energy, dark blue indicates the lowest impact energy. No on-axis charge is assumed.
This also illustrates why $\mathrm{H}^{+}$ions starting at rest and with some thermal velocity distribution show very little difference in their trajectories; the ions quickly accelerate up to many times the thermal velocity. Tests were done assuming a distribution of starting energies based on $k_{B} T$, but no significant impact upon the particle tracks were found.

Finally, the impact energy distribution on the cathode is determined. The impact energy plots in Figs. 4 and 5 illustrate only which particle starting locations result in ions impacting the cathode plane; it does not show where the ions deposit their energy on the cathode. Because the particle starting locations are tracked, we may histogram the impact energy based on all ions started either within a given radius (e.g., to model the ion generation of an electron beam with uniform transverse distribution) or within a given cylindrical shell (e.g., to help model the effects of an electron beam with a nonuniform transverse distribution).

Figure 6(a) shows the impact energy histogram on the cathode plane for a $1-\mathrm{cm}$ radius about the axis of the gun, assuming that no on-axis charge is present; Fig. 6(b) assumes an on-axis charge equivalent to $1 \mathrm{nC}$ per bunch, every bucket filled. The histogram bin widths are scaled to represent equal area rings on the cathode plane, and the vertical axis is the fractional energy deposited within the ring, normalized to the total energy deposited over the entire back plane of the gun. In both figures the red curves include only ions generated within $0.5 \mathrm{~cm}$ of the gun's axis, while the blue curves include all ion starting locations. The presence of a high beam current is seen to be beneficial in that it provides, in effect, a strongly overfocusing lens that acts to diffuse the hydrogen ion current over a larger area of the cathode; the distribution is still peaked in the center of the cathode, as expected, however.

The actual energy (or power) deposited on the cathode depends on the partial pressure of hydrogen within the gun (or molecular species from which it may be liberated), the electron bunch charge, the number of electron bunches generated (or, for power calculations, the beam repetition rate), and the probability of ionization; a sample calculation is performed later.

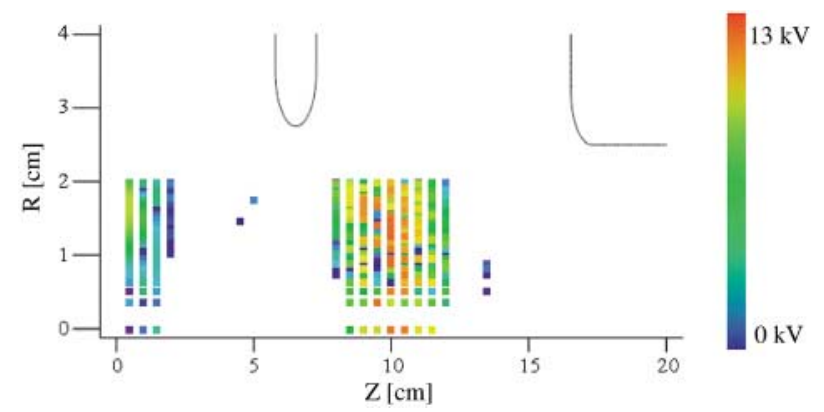

FIG. 5. (Color) Impact energy at the cathode plane as a function of starting location, for $\mathrm{H}^{+}$ions. Red indicates the highest impact energy, dark blue indicates no impact. On-axis electron charge is equivalent to $1 \mathrm{nC}$ per bunch, every bunch filled. 


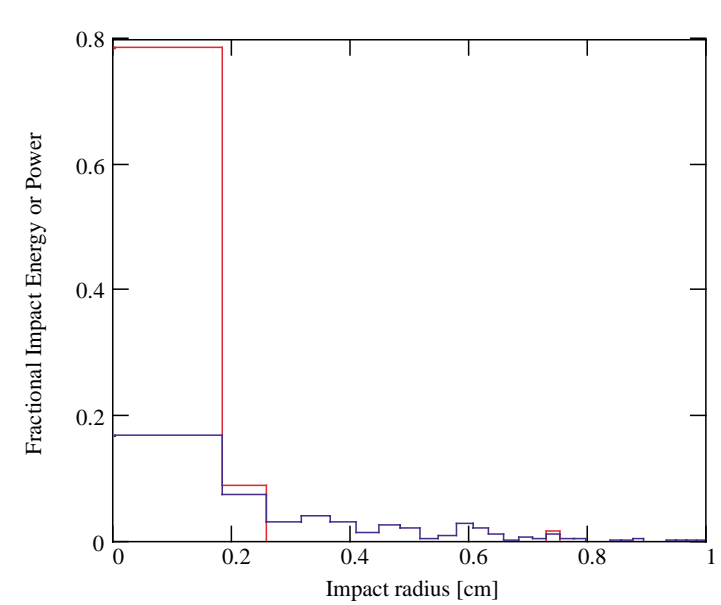

(a)

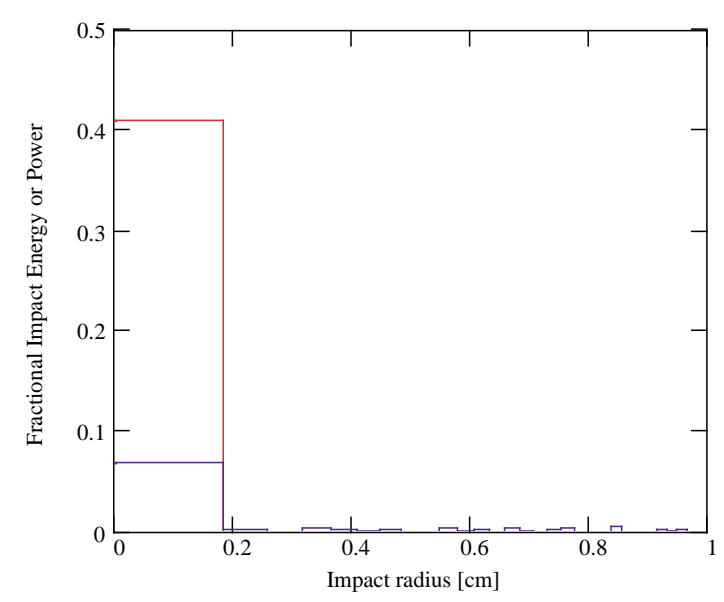

FIG. 6. (Color) Impact energy histogram for hydrogen ions generated within $0.5 \mathrm{~cm}$ of the gun axis (red), and for all ion starting locations (blue). (a) assumes no on-axis beam charge, (b) assumes 1-nC bunch charge, every bunch filled. The vertical axis is normalized to the total energy deposited to the cathode plate, for ions within the specified generating radius.

In any event, the ion flight paths and impact energy histograms indicate that the model $L$-band photoinjector is fairly good at funneling $\mathrm{H}^{+}$ions towards the cathode and that the ions can impact with significant energy. This alone indicates possible problems for long-pulse operation with high-QE cathodes, especially if a relatively low average current is generated. (In this context, "low" means that the ion motion is effectively the same as with no on-axis charge present.) Somewhat surprisingly, these results indicate that the use of higher beam currents may in some circumstances actually help to preserve cathode longevity by more uniformly distributing the ion impacts on the cathode plane. This would have to be balanced, however, against the greater rate of ion production from the continuous presence of the electron beam.

\section{Simulation results $-\mathrm{H}_{2} \mathrm{O}^{+}$ions}

Simulations were also performed using ions with mass equivalent to singly ionized water molecules. These simu- lations therefore include the assumptions that multiple ionization does not take place and that the water molecule is not disassociated due to repeated interactions with the electron beam, in addition to the other assumptions used for the hydrogen simulations. The ion starting positions and phases were selected in the same fashion as described above for $\mathrm{H}^{+}$ions.

Impact energy plots for water ions are shown in Fig. 7, which assumes no on-axis electron beam charge distribution, and Fig. 8, which assumes the nominal 1-nC charge per bunch, every bunch filled. An impact energy histogram over the cathode is shown in Fig. 9. There are very significant differences between the two impact energy plots, and the impact energy is in general much reduced from the case of $\mathrm{H}^{+}$ions presented above. For no on-axis charge, the impact energy histograms of $\mathrm{H}$ and $\mathrm{H}_{2} \mathrm{O}$ are very similar; in the presence of on-axis charge, however, the $\mathrm{H}_{2} \mathrm{O}$ impact energy histogram shows a much greater dispersion of the deposited energy across the cathode surface.

The reasons for these differences may be explained as follows. Consider both an $\mathrm{H}^{+}$ion and a water ion (approximately 18 times as massive) in the field of the gun. The field accelerates the particles and they begin to move; however, because of their mass the acceleration is very low, at least compared to an electron. One complete rf period later, the ion will have been induced to drift somewhat and, depending on the field uniformity at the starting location after one rf period, the ion may be returned to rest or may have acquired some drift velocity. The amount of drift velocity increase will depend on the local field uniformity. Therefore, one would expect the ions to gain most of their momentum in the regions where the rf field intensity has a large gradient with respect to position, e.g., near the nose cones. Examining the $\mathrm{H}^{+}$flight paths shown in Fig. 3, this is seen to be the case. All other things being equal, the more massive the ion, the smaller the distance it will move in a single rf period; therefore, it will acquire a smaller velocity kick. Thus, one would expect that hydrogen ions would pick up energy faster, and obtain a higher final energy, than heavier species. This is confirmed with the results of the $\mathrm{H}_{2} \mathrm{O}^{+}$ion tracking.

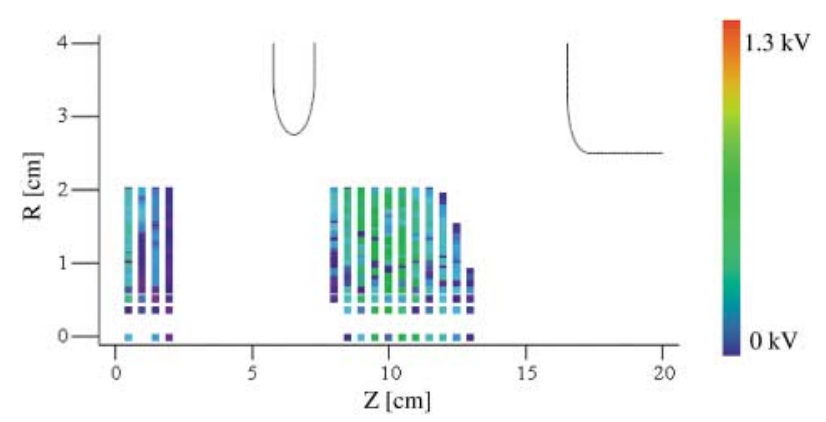

FIG. 7. (Color) Impact energy plot for $\mathrm{H}_{2} \mathrm{O}^{+}$ions, assuming no on-axis charge distribution. Note that the vertical scale is reduced 1 order of magnitude from the $\mathrm{H}^{+}$plots. 


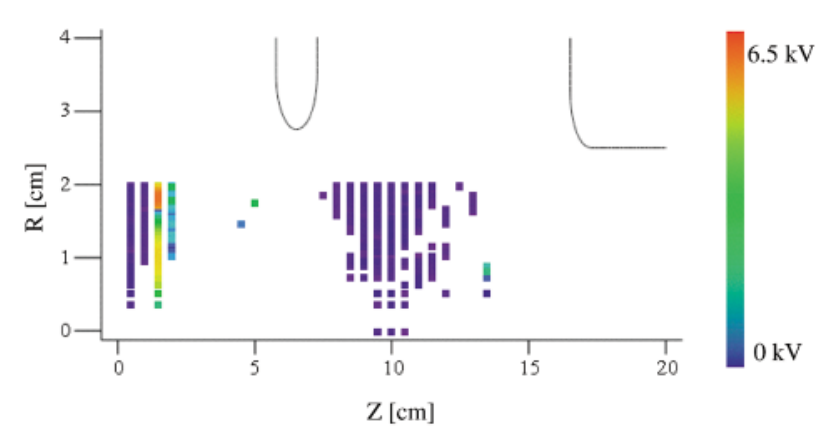

FIG. 8. Impact energy plot for $\mathrm{H}_{2} \mathrm{O}^{+}$, assuming a 1-nC bunch charge and every bucket filled. Note that the vertical scale differs from both the $\mathrm{H}^{+}$and that in Fig. 7 .

For this reason, the relative effect of the on-axis charge's fields should be greater for heavier ions than for lighter ions; the field from the on-axis electron beam is effectively a dc field (on the time scales of the ion motion) and therefore does not rely on a positional change and local field gradient to impart an acceleration. This is in fact the case observed for $\mathrm{H}_{2} \mathrm{O}^{+}$, where there is clearly

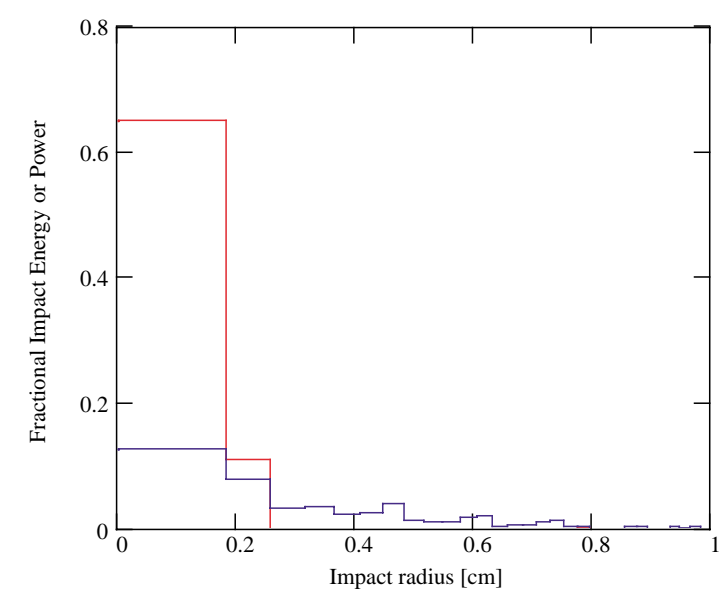

(a)

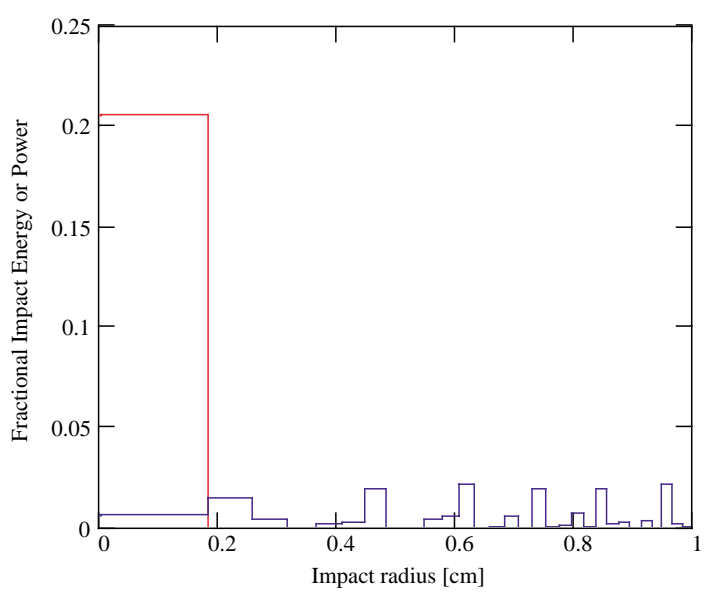

(b)

FIG. 9. (Color) Impact energy histogram for $\mathrm{H}_{2} \mathrm{O}^{+}$ions generated within $0.5 \mathrm{~cm}$ of the gun axis (red), and for all ion starting locations (blue). (a) assumes no on-axis beam charge, (b) assumes 1-nC bunch charge, every bunch filled. a strong change in the particle impact energy distribution and potential source points from the presence of the on-axis charge. One would not expect, however, the thermal distribution to play a greater role as the mass of the ion species increases because the starting velocity of the ions would decrease as the mass of the species is increased. This is in fact the case; as was the case for the $\mathrm{H}^{+}$ions, there is little obvious difference between the ion tracks of $\mathrm{H}_{2} \mathrm{O}^{+}$ions with and without an imposed thermal velocity distribution, based on a temperature of $300 \mathrm{~K}$.

\section{INTERPRETATION: HYDROGEN}

Finally, we use the results of the hydrogen simulations to determine, for the case of 1-nC charge per bunch and every bucket filled, the amount of hydrogen ions impacting on the cathode per second.

The ionization rate per unit volume for impact ionization is given by

$$
\frac{d n}{d t}=n_{b} n_{g} \sigma_{i} v
$$

where $n_{b}$ is the density of the electron beam, $n_{g}$ is the density of the gas species, $\sigma_{i}$ is the ionization cross section and is about $2 \times 10^{-23} \mathrm{~m}^{2}$ at the average electron beam energies in the gun, and $v$ is the beam velocity [12]. Expressed in terms of the total number of hydrogen ions created per rf period, this may be rewritten as

$$
\frac{d N}{d t}=\frac{Q_{b} P \sigma_{i} c}{e k_{B} T} D
$$

where $N$ is the total number of ions generated, $Q_{b}$ is the bunch charge, $P$ is the partial gas pressure, $\sigma_{i} \sim 2 \times$ $10^{-23} \mathrm{~m}^{2}, c$ is the speed of light, $e$ is the electron charge, $k_{B}$ is Boltzmann's constant, $T$ is the temperature in kelvin, and $D$ is the duty factor expressed in terms of the fraction of possible rf buckets filled. [Only impact ionization is considered. The electron beam radius and length are both large enough in the gun (on the order of several $\mathrm{mm}$ and $5-10 \mathrm{~mm}$, respectively) such that the beam self-fields are about 3 orders of magnitude below that required for tunneling ionization to occur during the time the beam is in the vicinity of a residual gas molecule [13].]

The electron beam density $n_{b}$ is taken as an average and is calculated by dividing the bunch charge by the volume swept out by the bunch in a single rf period. Note that the physical dimensions of the electron beam (e.g., radius) do not appear when expressed in this fashion. We can also calculate the number of ions generated per beam pulse as

$$
N_{p}=\frac{d N}{d t} \frac{\tau_{\mathrm{rf}}}{D}=\frac{Q_{b} P \sigma_{i} c}{e k_{b} T f_{\mathrm{rf}}},
$$

where $\tau_{\mathrm{rf}}$ is the $\mathrm{rf}$ period, and $f_{\mathrm{rf}}=1 / \tau_{\mathrm{rf}}$ is the resonant frequency of the cavity. Note that, if the bunch 
charge, gas pressure, or ionization cross section is low enough, this can result in an ion production rate of less than one per rf period. Note also that the rate of ion production scales inversely with temperature, meaning that, for the same pressure, superconducting if guns should have much higher rates of ion creation than normal-conducting rf guns.

Next, from the $\mathrm{H}^{+}$simulation with the on-axis charge distribution, we find that, of the ions generated within $0.5 \mathrm{~cm}$ of the bore, approximately $1 / 3$ will impact on the cathode. Using a 1-nC bunch charge, a duty factor of 1 (i.e., every bunch filled), and a partial pressure of hydrogen of $10^{-13}$ Torr, we obtain a hydrogen ion current to the cathode of $64 \mathrm{fA}$, assuming cw operation. The mean impact kinetic energy of these ions is approximately $7 \mathrm{kV}$, for a power delivery to the cathode of $0.5 \mathrm{nW}$. This is the total beam current delivered to the entire cathode surface; however, we know from Fig. 6(b) that roughly half of this current is going to be impacting close to the center of the cathode plate.

This is a low rate of energy delivery to the cathode and, based on the results for water ions, should be even lower for more massive species. The delivered current scales directly with the partial pressure of the species inside the gun. It also scales with the average electron beam current; however, as the beam current can have an influence on the ion trajectories, the exact scaling is more complex. This simple calculation also does not take into account the steep rise in ionization cross section for electron beam energies in the $10^{1}-10^{4} \mathrm{eV}$ range, which can be as high as $10^{-20} \mathrm{~m}^{2}$. This potentially represents a much larger source of ions in the vicinity of the cathode, which would immediately backstream towards the cathode. It should also be noted that, for the case of water ions, when an on-axis charge distribution was assumed, the ions with the greatest impact energy were generated in the vicinity of the cathode (see Fig. 8).

Based on the calculated ion current, the initial approximation of ignoring ion-ion and ion-beam interaction is probably reasonable, given the other approximations made. The simulations do, however, show evidence of ion trapping within the gun close to the nose cones between the cathode and full cells. With sufficient rf pulse duration and average beam current, one could conceive of an ion charge buildup large enough to have an impact on the electron beam and ion flow. For the beam and gas parameters listed above, and assuming that $10 \%$ of the ions generated are trapped, it would take approximately $86 \mathrm{~min}$ to generate and trap $100 \mathrm{pC}$ of hydrogen ions, assuming no deionization takes place, the generation and capture process is not adversely affected by the local buildup of space charge, and the assumptions regarding the $\mathrm{cw}$ electron beam approximation are valid. The computational requirements for approximately 1.5 simulated "real-time" hours are beyond the scope of this work.

\section{CONCLUSIONS AND FUTURE DIRECTIONS}

Long-pulse rf guns may experience deposition of energy upon their cathodes from lighter ionized species such as hydrogen. This is more true for high average beam current guns, where the ions will be continually generated and pumped towards the cathode. The actual ion current of a given species towards the cathode will depend on the partial pressure in the gun, the average beam current in the gun, and the ionization cross section; but, given these parameters, the above results can provide at least an estimate of the potential for damage. The ions resulting from the interaction between the relativistic electron beam and the residual gas do not result in a large current flow to the cathode; however, the much larger ionization cross section near the cathode surface (where the beam is under $0.1 \mathrm{MeV}$ in kinetic energy, for instance) may prove to be cause for concern, especially if the gun fields are good at transporting ions towards the cathode surface. This would require further study.

The methods used in this work are relatively straightforward and may be extended in any of several possible directions. For example, a more reasonable model of the electron beam propagation in the gun could be used to incorporate the time-varying electric and magnetic fields from the beam upon the ion flow. The possibility of multiple ionization events could also be incorporated, although this would require greater modifications to existing codes. Also, the electron beam energy gain profile could be used to better determine the ionization cross section at any point in the gun. At this point, however, it does not appear as though ion beam currents in long-pulse photocathode rf guns pose significant risks to cathode longevity as long as the residual species partial pressures, particularly of lighter species, are well controlled.

Clearly, there are many questions remaining and these conclusions need to be verified experimentally. Many of these questions could not be reasonably answered until a long-pulse photoinjector, incorporating a high-QE cathode, became available. One such gun was the Boeing FEL gun, mentioned above; however, that facility is in the process of being decommissioned. Other possibilities include the TESLA Test Facility rf gun, which operates with a nominal rf pulse duration of $0.8 \mathrm{~ms}$ [14], its sister gun at Fermi National Accelerator Laboratory, and the DESY-Zeuthen Photoinjector Test Facility.

\section{ACKNOWLEDGMENTS}

The author wishes to thank Manoel Conde and John Power at the Argonne Wakefield Accelerator for useful discussions and gun data files, Henry Freund of SAIC for timely input, and Lloyd Young and James Billen at Los Alamos for advice on setting up the simulations. This work is supported by the U.S. Department of Energy, Office of Basic Energy Science, under Contract No. W-31-109ENG-38. 
[1] Proceedings of the PERL Photocathode Gun Workshop, Brookhaven National Laboratory, 2001, edited by X.J. Wang (BNL Report No. BNL-52624, 2001) (http:// nslsweb.nsls.bnl.gov/nsls/org/PERL/).

[2] http://tesla.desy.de/new_pages/TDR_CD/start.html.

[3] T.D. Hayward et al., in Proceedings of the 1995 Particle Accelerator Conference, Dallas, Texas (IEEE, Piscataway, NJ, 1995) (http://accelconf.web.cern.ch/AccelConf/ p95/ARTICLES/FAA/FAA27.PDF).

[4] T. Siggins et al., http://www.fel.duke.edu/fel2000/ program/proceedings/MO-3-03.pdf.

[5] W. Hartung et al., http://pacwebserver.fnal.gov/papers/ Wednesday/AM_Poster/WPAH063.pdf.

[6] D.H. Dowell (private communication); S. Schreiber (private communication).

[7] As mentioned, there are several variations of the basic $\pi$-mode photoinjector. A good overview of the basics, with many references, is by J. E. Clendenin, http://linac96. web.cern.ch/Linac96/Proceedings/Tuesday/TU204/Paper. pdf.
[8] J. H. Billen and L. M. Young, Los Alamos National Laboratory Report No. LA-UR-96-1834, 2000.

[9] L. M. Young and J. H. Billen, Los Alamos National Laboratory Report No. LA-UR-96-1835, 2000.

[10] For more information on PARMELA and some of its derivatives, http://pbpl.physics.ucla.edu/docs/parmela.pdf may be of interest.

[11] Manoel Conde (private communication).

[12] Martin Reiser, in Theory and Design of Charged Particle Beams, edited by Mel Month (Wiley, New York, 1994), pp. 273-276.

[13] T.O. Raubenheimer and Pisin Chen, SLAC Report No. SLAC-PUB-5893, 2002.

[14] H. Weise, for the TESLA Collaboration, in Proceedings of the 1995 Particle Accelerator Conference, Dallas, Texas (IEEE, Piscataway, NJ, 1995) (http://accelconf.web.cern. ch/AccelConf/p95/ARTICLES/RPB/RPB08.PDF). 